\title{
Efficient Speech De-noising Applied to Colored Noise Based Dynamic Low-pass Filter Supervised by Cascade Neural Networks
}

\author{
Selmani Anissa, Seddik hassene, Mbarki zouhair \\ ESSTT, 5 Av. Taha hussien, 1008, Tunis, Tunisia \\ E-mails: anissaselmani02@gmail.com, hassene.seddik@est.rnu.tn
}

\begin{abstract}
In this paper, we investigated the enhancement of speech by applying an optimal adaptive low-pass filter supervised by neural network. The corruption of speech due to the presence of additive noise causes its degradation in quality and intelligibility.

To filter this distorted signal in its spatial representation is a hard task. This task is more difficult to realize if the distortion are caused by colored noise.

In addition using a static filter is not efficient due to the speech signal variability. In the same sentence a phoneme can change in shape and amplitude. For these constraints, we propose to apply a low-pass filter with Gaussian core supervised by neural networks. Filtering strength changes continuously with the phoneme variation to generate a variable filter that change over the whole sentence.
\end{abstract}

Keywords - Speech de-noising, neural networks, optimization, Gaussian filter, parameter estimation

\section{INTRODUCTION}

De-noising speech signals is one of the main problems that should be solved for telecommunication systems and humain machine communication.

It has been an area of active research and continues to trigger the researchers' attention in various fields, for example, [2], [3], [4], [5] and [6]. The presence of background noise in speech significantly reduces the intelligibility of speech. Degradation of speech severely affects a person's ability, whether impaired or normal hearing, to understand what the speaker is saying [7]. Recent researches have developed a variety of theoretical methods to fight this problem, but the complexity of this task is always linked to the random nature of the noise and the inherent complexities of speech. Another important problem in speech enhancement is the phoneme distinction [8]. Noise does not impact equally on all phonemes because of the distinct acoustic properties of each phoneme. One key to speech enhancement is satisfactorily modeling the human speech production process. Such modeling is difficult because speech signals are, in general, highly non stationary. Classical speech de-noising methods such as wavelet de-noising [9], Spectral subtract de-noising [10], Kalman filtering [11], and de-noising by a static Gaussian filter is not very upgrading. This paper presents a method for speech enhancement based on a dynamic smart Gaussian filter. Our aims is to remove real- colored noise which have, unlike white Gaussian noise (WGN), a spectrum that is not flat [7]. Therefore, the noise does not affect the speech signal uniformly over the whole spectrum.
Some frequencies are affected more adversely than others. This paper is an extension of our previous works that focuses on the use of a dynamic Gaussian filter for white noise removal [1]. The paper is arranged as follows. Firstly, we describe Gaussian filtering and problems related to its statics character in section 2. Then, we build theory in Section 3 which allows identification of an optimal oopt and we explain the principle of our approach. We show experimentally how the identification of the smart dynamic Gaussian filter can be found in Section 4 and conclude in Section 5.

\section{PROBLEM STATEMENT}

\subsection{GAUSSIAN FILTER}

In the time domain, a Gaussian filter is parameterized by its means $\mu$ and variance $\sigma 2$. The choice of the Gaussian is very important to assess the quality of the de-noising process. But this method has some drawbacks since this filter size depends directly on the standard deviation of the Gaussian $\sigma$, which controls its power and flattening, so, it determines the amount of smoothing. To a certain value of sigma, the Gaussian filter is capable to filter the signal by removing noise without destroying it. But, if we increase sigma, the cutoff frequency and the Gaussian filter's bandwidth will decrease and eventually will lead to eliminate more noise but also the possibility of signal distortion may increase. In the limiting case when $\sigma$ tends to 0 , we have an all pass filter which affects neither the signal nor the noise. As we increase $\sigma$ the out of band noise is filtered without any distortion to the signal until the value of $\sigma$ is such that it not only removes the out of band noise but also distorts the signal [12]. We can therefore conclude that we must design a dynamic Gaussian filter that varies depending on two essential criteria:

- Phoneme variability in amplitude and shape

- Noise intensity variation

The mathematical representation of the Gaussian is described as follows:

$G\left(\mu, \sigma^{2}, t\right)=\frac{1}{\sqrt{2 \pi \sigma^{2}}} \exp \left[\frac{-(t-\mu)^{2}}{2 \sigma^{2}}\right]$ 


\subsection{CHARACTERISTICS OF THE SPEECH SIGANL}

The speech signal is a set of words; these words are also made of phonemes which have different characteristics. Vowels, for example, have dominant frequency components at harmonic locations, especially at the formant locations. More aggressive noise over-subtraction can be performed on voiced segments without degrading audio quality. On the other hand, the spectral distribution of unvoiced sounds, like unvoiced plosive and fricative, is very similar to that of white noise. The original components are thus obscured and subsequent aggressive noise suppression results in severe signal distortion [8].
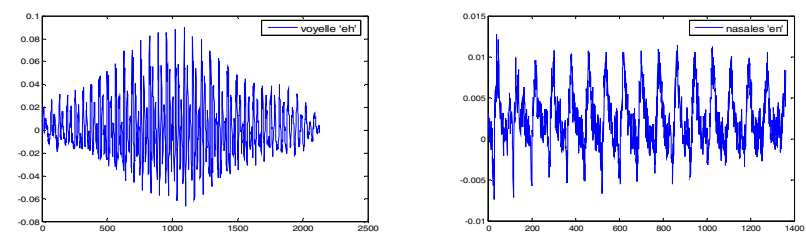

Fig.2 Examples of TIMIT phonemes database

Therefore, Syllable segmentation is an important access to extract audio structure and content and is a basis for further audio retrieval and analysis [13].

It is theoretically and experimentally demonstrated that there are highly nonlinear phenomena present in the process of speech production [14-16].

However, the traditional approach to speech de-noising is based on linear techniques that can not sufficiently take into account the complex dynamics of speech.

Therefore, it is clear that to achieve more complex functions of nonlinear filtering; it is natural to use an artificial neural network for parameter estimation.

\section{THE PROPOSED APPROACH:}

We have used a speech database (TIMIT) constituted by 6300 sentences pronounced in American English by 630 speakers (male and female. We will work with a speech signal recorded at 16000).

First step: This sentence is corrupted by a colored noise.

Second step: Phonemic segmentation.

Third step: Each phoneme is filtered several times by a Gaussian static filter with different variance to obtain the optimal value of $\sigma$ which determines the amplitude and the support size.

Fourth step: Neural network training with noised phonemes as inputs and optimal computed variance as constraint.

Fifth step: Neuronal network's test

Sixth step: Outputs recovery relative to new inputs.
Seventh step: Filtering phonemes by sigma values obtained at the output of network.

Eighth step: Computing two distortions measures SNR and PESQ.

\subsection{COLORED NOISE:}

Real world noise is mostly colored and does not affect the speech signal uniformly over the entire spectrum. Image 4 illustrates the original sentence and the sentence corrupted by colored pink Gaussian noise:
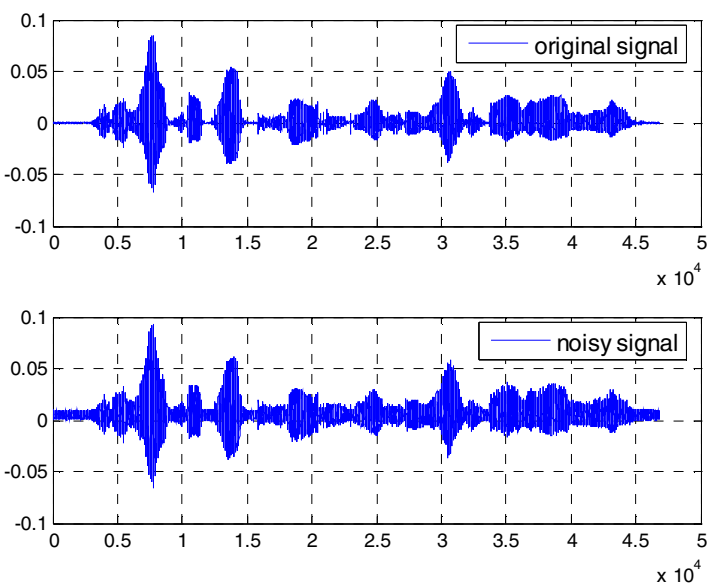

Fig. 3 original and noisy speech signal

\subsection{PARAMETERS OPTIMIZATION:}

In this step, we should make the choice of optimal variance that generates the best SNR after being filtered for each phoneme. The following figure shows the SNR function varying with $\sigma$. We convolve each phoneme iteratively with a 1000 of Gaussian filters. In each iteration the $\sigma$ value is changed with a variation $\mathrm{d} \sigma=0.01$.

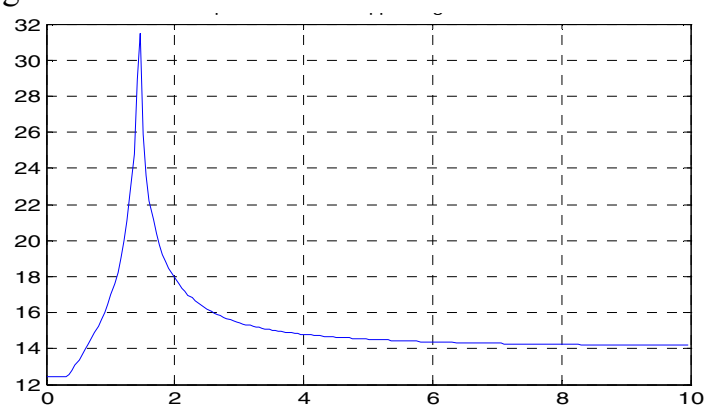

Fig. $4 \mathrm{SNR}=\mathrm{f}(\sigma)$

From this figure we are able to determinate accurately the optimal sigma's value called $\sigma_{\text {opt }}$.

$S N R=f(\sigma)$

$f(\sigma)=\sum_{i=d \sigma}^{N} x(n) * h_{i}(\alpha-n, \sigma)$

Where: $\mathrm{N}=8$ and $d \sigma=0.01$ 
$\frac{d f(\sigma)}{d \sigma}=0 \Rightarrow \frac{d S N R(\sigma)}{d \sigma} \Rightarrow \sigma_{o p t}=\max (\operatorname{SNR})$

The optimal standard deviation is obtained as shown by equation 4 and figure 4 .

\subsection{NEURAL NETWORKS}

Neural networks are already widely exploited in the classification areas and pattern recognition. The parallel architecture used in our algorithm is able to learn efficiently the introduced inputs that are divided in clusters of 6 phonemes each one. While the colored noise is more difficult to remove and the length of the phonemes is very high this architecture is found to be able de learn the entrees and generate correct outputs. The Back-Propagation architecture is used. The outputs are computed as follows:

$o(x)=f\left(w_{0}+\sum_{i=1}^{n} w_{i} x_{i}\right)=f\left(w_{0}+w^{T} x\right)$

In our case:

$$
\begin{aligned}
o[x(n)] & =f\left(w_{R N}+\sum_{i=1}^{N} w_{i} x_{i}\right) \\
& =f\left(w_{R N}+w^{T} x[n]\right)=\sigma_{R N T}
\end{aligned}
$$

Where wRN denotes the learned weights, $\mathrm{w}=(\mathrm{w} 1, \ldots, \mathrm{wn})$ and $\mathrm{x}[\mathrm{n}]=(\mathrm{x} 1, \ldots, \mathrm{xn})$ is the input.

Once the activities of all output units have been determined, the network computes the quadratic error E (equation 7), which is defined by the equation (8) in our case:

$$
E=\frac{1}{2} \sum_{i=1}^{k}(Y i-d i)^{2}
$$

$$
E_{\sigma}=\frac{1}{2} \sum_{i=1}^{k}\left(\sigma_{o p t}-\sigma_{R N}\right)^{2}
$$

Where yi is the activity level of the $\mathrm{i}^{\text {th }}$ unit in the top layer and $\mathrm{di}$ is the desired output of the $\mathrm{i}^{\text {th }}$ unit. The variable Gaussian function becomes:

$G\left(t, \sigma_{R N T}\right)=\frac{1}{\sqrt{2 \pi \sigma_{R N T}^{2}}} \exp \left(\frac{-t^{2}}{2 \sigma_{R N T}^{2}}\right)$

Many structures of neural networks are tested. Non linear activation function is applied on all neurons due to the non linear behavior of the processed signal. While the used colored noise is near to real noise and difficult to remove, the choice of a cascade networks is considered. Every network is composed by only three layers "input layer, hidden layer and output layer". Every group of phonemes composed by three or four voiced phoneme are noised and learned by a network. A thresholding system gather all the outputs and distribute them over the noised speech signal.

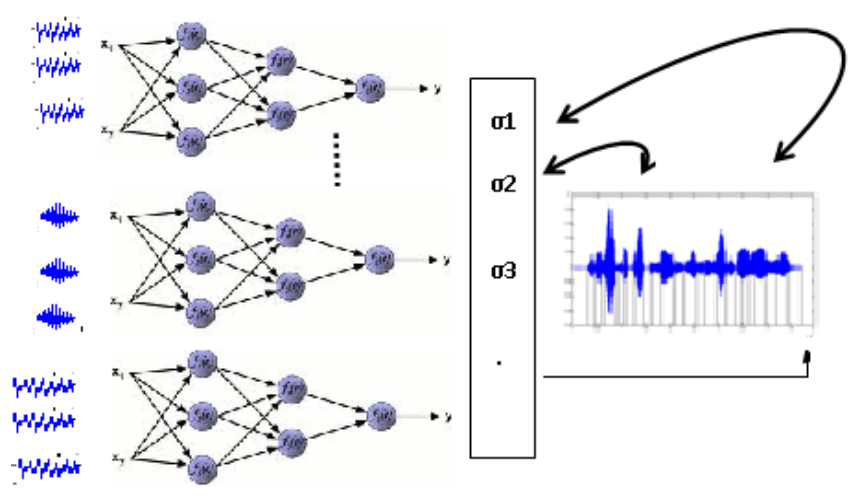

Fig.5 Neuronal networks parallel architecture

\section{RESULTS AND DISCUSSION:}

To evaluate the performance of the approach, we will use a criterion widely used in simulation tests, which is the signal to noise ratio (SNR) of the reconstructed signal which is given by:

$$
\operatorname{SNR}(d b)=10 \log \frac{\sum_{i=1}^{N}|x(i)|^{2}}{\sum_{i=1}^{N}|x(i)-\hat{x}(i)|^{2}}
$$

The obtained values of the SNR after de-noising voiced phoneme are reported in the following figure.

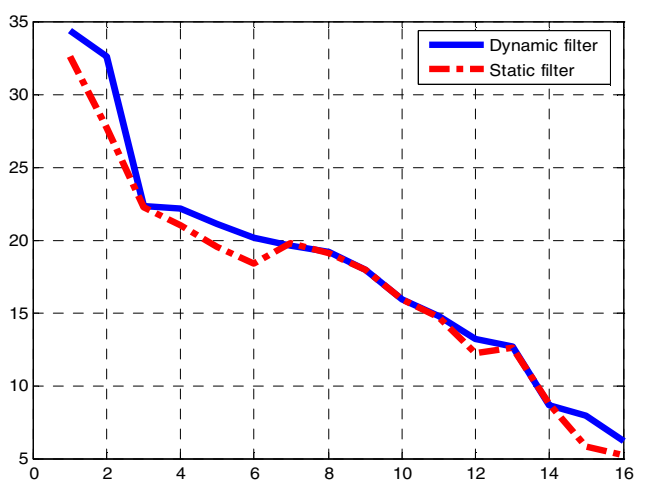

Fig.6 SNR of the voiced phonemes

Shown from the test results, compared with traditional denoising by static Gaussian filter, the average SNR has been improved a certain extent through Dynamic Gaussian filtering. This method can effectively reduce noise and meet the needs of practical applications for voiced phoneme. The obtained values of the signal to noise ratio after de-noising unvoiced phoneme are reported in the next figure. 


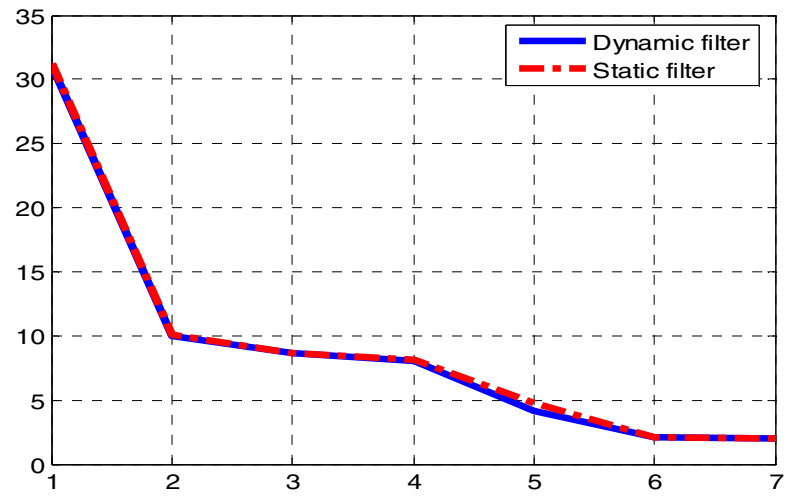

Fig.7 SNR of the unvoiced phonemes

Other criteria considered for assessing the performance of our approach, is the perceptual evaluation of speech quality PESQ. This method is essentially based on the most commonly used measure MOS (Mean Opinion Score). The MOS is the result of analysis by absolute categories ACR (Absolute Category Rating) in which a group of listeners hear a set of audio files and assesses independently, one by one, according to a rating scale of perceived quality.

TAB.1: PESQ RATE

\begin{tabular}{|c|c|}
\hline MOS Score & MOS Quality \\
\hline 5 & excellent \\
\hline 4 & good \\
\hline 3 & Passable \\
\hline 2 & bad \\
\hline 1 & Very bad \\
\hline
\end{tabular}

The obtained values of PESQ evaluation after de-noising voiced phoneme are reported in the next table.

TAB.2: PESQ RATE FOR VOICED PHONEMES

\begin{tabular}{|c|c|c|}
\hline PESQ & $\begin{array}{c}\text { Enhanced speech } \\
\text { with RN parameters }\end{array}$ & $\begin{array}{c}\text { Enhanced speech } \\
\text { with static filter }\end{array}$ \\
\hline Phoneme & & 2.805 \\
\hline ix & 3.982 & 4.160 \\
\hline hv & 4.150 & 4.440 \\
\hline eh & 4.445 & 3.320 \\
\hline ih & 3.334 & 4.233 \\
\hline ah & 4.232 & 4.324 \\
\hline ux & 4.361 & 4.275 \\
\hline q & 4.275 & 3.983 \\
\hline en & 4.233 & 3.793 \\
\hline s & 3.794 & 4.187 \\
\hline w & 4.257 & 4.337 \\
\hline ao & 4.348 & 3.956 \\
\hline y & 3.955 & \\
\hline
\end{tabular}

The PESQ evaluation after de-noising unvoiced phoneme is reported in the following table.
TAB.3: PESQ RATE FOR UN-VOICED PHONEMES

\begin{tabular}{|c|c|c|}
\hline PESQ & $\begin{array}{c}\text { Enhanced speech } \\
\text { with RN parameters }\end{array}$ & $\begin{array}{c}\text { Enhanced speech } \\
\text { with static filter }\end{array}$ \\
\cline { 1 - 1 } Phoneme & & \\
\hline Sh & 3.989 & 3.997 \\
\hline jh & 3.320 & 3.334 \\
\hline Dcl & 2.245 & 1.749 \\
\hline Kcl & 1.920 & 1.920 \\
\hline sl+kl & 3.800 & 3.800 \\
\hline Ao+l & 4.362 & 4.380 \\
\hline other phonemes & 3.896 & 3.985 \\
\hline
\end{tabular}

From the simulation results, we can see that the performance of the proposed dynamic method is generally better than the conventional static filtering except the SNR measure in the case of unvoiced phoneme; which is normal views the characteristics of the unvoiced phoneme.

In fact, informal subjective listening tests confirm the SNR and the perceptual evaluation of speech quality PESQ values. Figure 7 and 8 represent the evolution of respectively noised and enhanced speech signal (voiced and unvoiced phoneme) using static and dynamic de-noising methods.
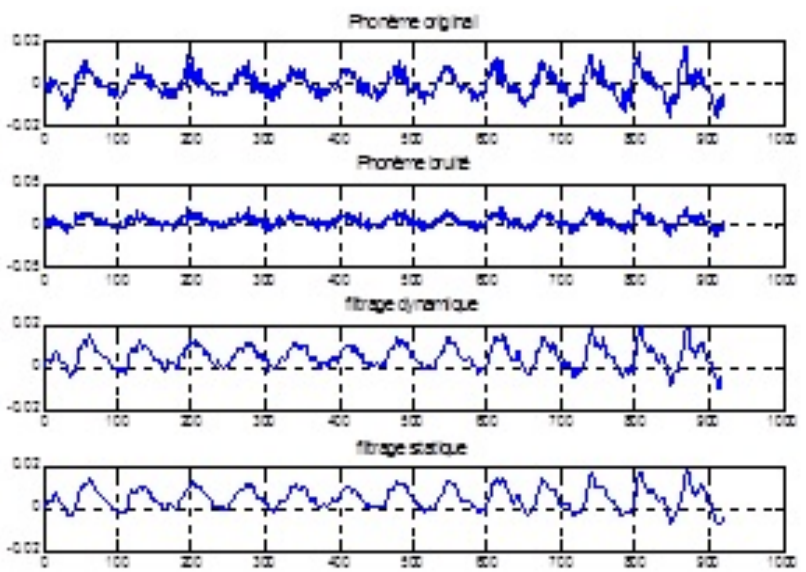

Fig.8 De-noising voiced phoneme « hv »
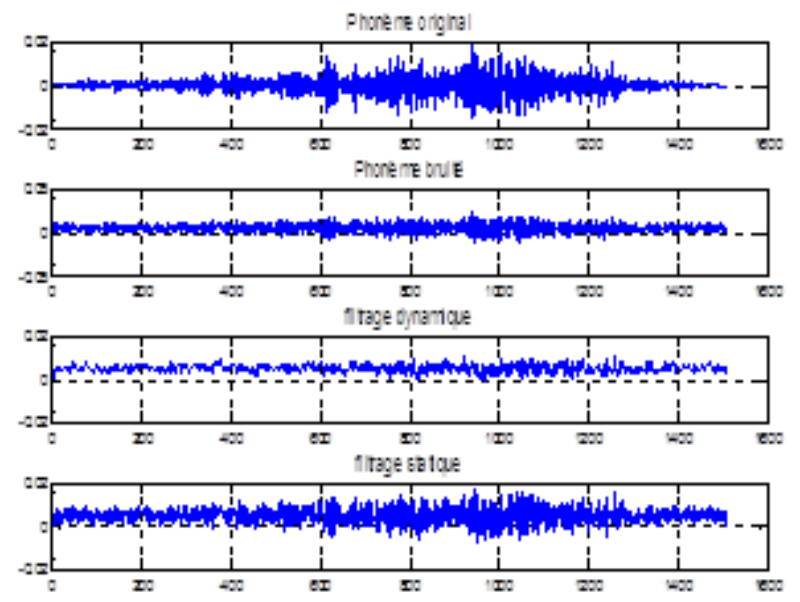

Fig.9 De-noising unvoiced phoneme « sh » 


\section{CONCLUSION}

In this paper, a new method for speech de-noising in the temporal representation is proposed. Based on neural network with cascade structure, a static Gaussian low-pass filter is converted to dynamic variable filter that changes with the phoneme allure and noise density and generates the optimal de-noising process. The simulation results proved that even with colored real noise, good results are obtained and the noise is always filtered without damaging the intelligibility of the speech signal. Different distortions measurements are applied and a comparison study with Gaussian filter with static shape is carried out.

\section{References:}

[1] A.Selmani, H.Seddik, "Conception d'un filtre Gaussien dynamique a optimisation neuronale pour le filtrage audio a temps réel", CRATT, Tunisia 2012.

[2] D. Crisan, M. Kouritzin, and J. Xiong, "Nonlinear filtering with signal dependent observation noise”, Electronic Journal of Probability, 2009, Vol:14

[3] R. Oktem, K. Egiazarian, V.V. Lukin, N.N. Ponomarenko, and O. V. Tsymbal, "Locally adaptive DCT filtering for signal-dependent noise removal”, Eurasip Journal on Advances in Signal Processing, 2007.Vol.2007.

[4] A. Buades,A. Silva, and B. S. Santos, "On image de-noising methods," Journal of digital imaging the official journal of the Society for Computer Applications in Radiology, 2010, Vol:4, no. 2, pp. $1-40$.

[5] C. Huang, H. Wang, and B. Long, "Signal de-noising based on emd," 2009 IEEE Circuits and Systems International Conference on Testing and Diagnosis, 2009 ,Vol. 1, no. 1, pp. 1-4.

[6] Y. Yang and Y. Wei, "Random interpolation average for signal denoising, ” IET Signal Processing, 2010,Vol:4, no. 6, p. 708.

[7] M. Mathel, Siva Prasad Nandyala2, Speech Enhancement Using Kalman Filter for white, random and color noise”. IEEE International Conference on Devices, Circuits and Systems (ICDCS), 15-16 March 2012. Vol:4.

[8] J M.E. Deidher, and A.S, "Spanias HMM-based speech enhancement using harmonic modeling”, Proceedings of IEEE ICASSP'97, Munich, Germany, 1997, pp.1175-1179.Vol:2.

[9] S.MANIKA NDAN,Asst Prof,Department of Ece, "Speech enhancement based on wavelet denoising”, Academic Open Internet Journal,2006

[10] M. Talbi, H. Belgacem, and A. Cherif, "A Wavelet Packet Speech Denoising using Spectral Entropy”, Tunisia,2007,

[11] J.D. Gibson, B. Koo, and S.D. Gray, "Filtering of Colored Noise for Speech Enhancement and Coding," IEEE Trans. Signal Processing, Aug.199, Vol. 39, No. 8, pp. 1732-1742.

[12] Sunil Kumar.K and M.Satish "Identifying Optimal Gaussian Filter for Gaussian Noise Removal", Third National Conference on Computer Vision, Pattern Recognition, Image Processing and Graphics, TCS Innovation Labs - Mumbai, Yantra Park, Thane (West), Maharastra, INDIA., Dec.2011

[13] Xi'an,China, "Speech denoising and Syllable segmentation based on Fractal dimension PAN Feng," University, International Conference on Measuring Technology and Mechatronics Automation 2010.Vol:3.

[14] A.Barney, C.Shadle, et P. Davies. "Fluid flow in a dynamical mechanical model of the vocal folds and tract", part $1 \& 2 . J$. Acoust. Soc. Amer, , (11 pages), Issue 1, pp. 456-466, Nov 1999; Vol:105.

[15] I. Kokkinos et P. Maragos, "Nonlinear speech analysis using models for chaotic systems". IEEE Transactions on Speech and Audio Processing", Vol. 13.NO. 6, NOVEMBER 2005.

[16] M.A.Little. "Biomechanically Informed Nonlinear Speech Signal Processing”. Thesis Doctorate, Oxford University, 2007. 